\title{
Anti-VEGF and anti-EGFR agents open up new horizons changing the landscape for their associations
}

\author{
Gerard Milano1
}

Received: 2 June 2016 / Accepted: 7 June 2016 / Published online: 16 June 2016

(C) The Author(s) 2016. This article is published with open access at Springerlink.com

\section{To the Editor}

Zaniboni and Formica recently published a review article considering preclinical and clinical data in the current context of optimal therapeutic sequences in metastatic colorectal cancer [1]. Their conclusions supported anti-EGFR agents as first-line treatment before anti-VEGF agents. Different biological and molecular treatment-conferred changes were advocated to support this order of drug combination. Surprisingly, an important aspect which was not considered by the authors is the current reconsideration of anti-VEGF and anti-EGFR effects through their respective and complementary abilities to modulate tumor immunity both directly and indirectly. For instance, on the one hand, involvement of $\mathrm{T}$ cells has been reported to be a component of the antitumor activity of EGFR-targeted monoclonal antibodies [2]. Also, EGFR activation has recently been shown to upregulate PDL-1 [3], potentially implying that EGFR inhibition can restore anti-tumor immunity. On the other hand, apart from its pro-angiogenic effect, VEGF must also be considered to be a strong immunosuppressor agent [4] and VEGF-targeted therapy has been shown very recently to combine favorably with checkpoint inhibitors based on pronounced $\mathrm{CD}^{+} \mathrm{T}$ cells tumor infiltration boosted by the anti-angiogenic drug [5].

Taken all together, these different features must lead us to reconsider anti-VEGF and anti-EGFR combinations, focusing not only on the association but also on the prospect of perspective for combinations with immunomodulatory agents like checkpoint inhibitors.

Open Access This article is distributed under the terms of the Creative Commons Attribution 4.0 International License (http://creativecommons.org/licenses/by/4.0/), which permits unrestricted use, distribution, and reproduction in any medium, provided you give appropriate credit to the original author(s) and the source, provide a link to the Creative Commons license, and indicate if changes were made.

\section{References}

1. Zaniboni A, Formica V (2016) The Best. First. Anti-EGFR before anti-VEGF, in the first-line treatment of RAS wild-type metastatic colorectal cancer: from bench to bedside. Cancer Chemother Pharmacol. doi:10.1007/s00280-016-3032-8

2. Yang X, Zhang X, Mortenson ED et al (2013) Cetuximab-mediated tumor regression depends on innate and adaptive immune responses. Mol Ther 21:91-100

3. Chen N, Fang W, Zhan J et al (2015) Upregulation of PD-L1 by EGFR activation mediates the immune escape in EGFR-driven NSCLC: implication for optional immune targeted therapy for NSCLC patients with EGFR mutation. J Thoracic Oncol 10:910-923

4. Motz GT, Coukos G (2011) The parallel lives of angiogenesis and immunosuppression: cancer and other tales. Nat Rev Immunol 11:702-711

5. Hodi FS, Lawrence D, Lezcano C et al (2014) Bevacizumab plus ipilimumab in patients with metastatic melanoma. Cancer Immunol Res 2:923-931
Gerard Milano

gerard.milano@nice.unicancer.fr

1 Nice, France 